\title{
MINERALIZATION OF OXIDIZED ALGINATE- GELATIN-BIPHASIC CALCIUM PHOSPHATE HYDROGEL COMPOSITE FOR BONE REGENERATION
}

\section{HANG DANG THI LE ${ }^{1,2}$, DUONG VAN THUY ${ }^{2}$, MINH DUNG TRUONG ${ }^{3}$, QUYEN TRAN NGOC ${ }^{1}$, HA TRAN BAO LE ${ }^{4}$, HOA NGUYEN KHANH ${ }^{5}$, KHOA NGUYEN CUU ${ }^{1}$ and PHUONG NGUYEN THI ${ }^{1}$}

${ }^{1}$ Institute of Applied Materials Science

Vietnam Academy Science and Technology

01 Mac Dinh Chi, District 1

Ho Chi Minh City

Vietnam

e-mail: ntphuongvc@yahoo.com.vn

International University

Ho Chi Minh City

Vietnam

${ }^{3}$ Department of Molecular Science and Technology

Ajou University

Suwon

Korea

${ }^{4}$ University of Sciences

Ho Chi Minh City

Vietnam

5Pham Ngoc Thach Medicine University

Ho Chi Minh City

Vietnam

Keywords and phrases: gelatin, oxidized alginate, BCP, hydrogel composite.

Received July 6, 2016 


\begin{abstract}
In this study, hydrogel composites based biphasic calcium phosphate (BCP), gelatin, and oxidized alginate were fabricated by Schiff-base reaction. Nanoparticles BCP were loaded into hydrogel to improve the ability of biomineralization and cell adhesion. The formation of apatite from hydrogel composites in stimulated body fluid (SBF) were characterized by energydispersive X-ray spectroscopy (EDS) and X-ray diffraction (XRD). In vitro study with bone marrow mesenchymal stem cells (MSCs) evidenced that cells were well-attached on hydrogel composites. Moreover, micro-CT data showed that hydrogel composites have bone surface/volume ratio and bone surface density higher than hydrogel. The obtained results indicated that the hydrogel composite could be a potential material for bone regeneration.
\end{abstract}

\title{
1. Introduction
}

Bone which is a rigid organ of vertebrates, provide a mechanical structure, protect the vital organs as well as support a reservoir of minerals for balance metabolism. Despite being capable of selfregeneration, some severe defects and/or physiological states making bone cannot heal by itself; surgical interventions using tremendous growth in the use of bone graft (i.e., autograft or allograft) provide the defect site of initial structural stability and osteogenic environment. While autograft and allograft of bone tissue are conventionally familiar as 'gold standard' treatment for bone loss and non-union defect, they remain some obvious limitations such as bone supply, donor site morbidity, pathogen transfer, and contour irregularities. In order to alleviate the aforementioned drawbacks induced by the traditional bone graft, some biosynthetic scaffolds for bone healing applications have been introduced [1].

One of the approaches in tissue engineering is hydrogel scaffold which involves three dimensional crosslinked hydrophilic polymeric networks. The process of crosslinking during synthesis creates a structural framework containing micro-cavities filled with water. Along with their unique features such as water uptake ability, space for drug or nutrient storage, cell immobilization ability, creation of microenvironment 
for cell culturing allowing nutrients, and metabolites to diffuse to and from the cells etc., hydrogels are being utilized for a wide range of applications. Many studies have shown that the hydrogel scaffolds are excellent bioactivity such as cell attachment and nontoxic degradation in vivo by tissue regeneration. Advanced tissue repairing approaches based on applying hydrogels not only give 3D structural support, but also interact with local cells to conduct several desired biological functions such as adhesion, spreading, proliferation, and matrix production [2].

Almost hydrogel systems lack the capacity to mineralize, which limit their application as bone substitutes. The concept of combining a hydrogel with an inorganic phase is inspired by the composite nature of bone itself [3]. There are many advantages of adding an inorganic phase such as providing nucleation for apatite layer formation, providing cell adhesion sites for enable integration with surrounding bone tissue $[4,5]$. The bio-mineralization is essentially required in many applications as a hard tissue implant due to forming chemical bonds of living bone throughout the bone like apatite and implant.

Calcium phosphate exhibits osteogenic property both in vitro and in vivo which makes them prominent material for future clinical applications as a hard tissue implant. Calcium phosphate has the advantages for bone tissue engineering applications over other conventional non-degradable bioceramics such as $\mathrm{TiO}_{2}, \mathrm{Al}_{2} \mathrm{O}_{3}$, and $\mathrm{ZrO}_{2}$. Firstly, chemical reactions on the surface strongly bond with native bones by means of a hydroxyl carbonate apatite (HCA) layer. Secondly, the released ions and dissolution products from calcium phosphate essentially benefit bone regeneration. Among calcium phosphate ceramics, such as hydroxyapatite (HAp) and TCP (tricalcium phosphate), osteoinductivity of BCP (HAp and TCP) has been reported as more efficient than HAp alone for repair of periodontal defects, and having better osteoinduction than single phasic HAp or TCP. HAp is stable in body fluid, while TCP is rather soluble. On the other hand, the 
dissolution rate of HAp in human body after implantation is too low to achieve the optimal results while the dissolution rate of TCP is too fast for bone bonding. Therefore, biphasic calcium phosphate, the combination of HAp, TCP may present a scope for optimal biodegradable calcium phosphates, were developed. For these materials constituted of HAp and TCP mixture, the adjustment of $\mathrm{Ca} / \mathrm{P}$ ratio value allows controlling the resorption rate. The combination of HAp, TCP can improve the mechanical properties, induce the proper biodegradation and promote the osteointegration that is increasing the bioactivity [6-9].

Natural hydrogels have some advantages such as their biocompatibility and biodegradability. Gelatin promotes cell adhesions because it contains integrin binding sites. Alginate is a biodegradable polysaccharide that can mimic the extracellular matrix. Oxidized alginate has gelling ability via the Schiff-base reaction with gelatin. Altogether, BCP, oxidized alginate and gelatin can be used to create the hydrogel composite that mimic natural living bone structure which mainly composed of nonstoichiometric hydroxyapatite and collagen fiber matrix [10-12].

Previously, the composite hydrogel systems consisting of oxidized alginate, gelatin, and biphasic calcium phosphate were developed in laboratory. Oxidized alginate-gelatin-BCP hydrogels showed an initial structural stability, a favorable environment for bone in growth and possibly biodegradation [13].

Extension the preceding study, the oxidized alginate-gelatin hydrogels combined varied BCP particles so as to improve the ability of biomineralization and cell adhesion is disclosed herein. Then, in vitro experiments with bone marrow mesenchymal stem cells (MSCs), and implanted the substitute to demonstrate its functionality in vivo. 


\section{Materials}

Sodium alginate (medium viscosity grade, viscosity of $2 \%$ solution $3500 \mathrm{cps}$ at $25^{\circ} \mathrm{C}$ ), gelatin from porcine skin (Bloom 300, Type A, MW 100,000), sodium metaperiodate were obtained from Sigma-Aldrich, USA. BCP powder was synthesized by using a microwave assisted process with a particle size of around $90-100 \mathrm{~nm}$ and the raw materials for it were calcium chloride $\left(\mathrm{CaCl}_{2}\right)$, di-sodium hydrogen phosphate $\left(\mathrm{Na}_{2} \mathrm{HPO}_{4}\right)$ were purchased from Merck. For the in vitro study, dimethylsulphoxide 99.0\% (DMSO) was purchased from Samchun Pure Chemical Co. Ltd, Korea. 3-[4,5-dimethylthiazol-2-yl]-2,5 diphenyltetrazolium bromide (MTT) solution were purchased from GibcoCarlsbad, CA. Phosphate buffered salinetablets (PBS) were obtained from Amresco, Korea. Mesenchymal stem cells (MSCs) derived from rabbit bone marrow.

\section{Methods}

\section{Oxidation of sodium alginate}

Sodium metaperiodate was dissolved in distilled water, and sodium alginate into ethanol; they were then stirred together for 24 hours in the dark at $25^{\circ} \mathrm{C}$. After sufficient time intervals, ethylene glycol was added to stop the oxidation reaction. The solution then dialyzed (MWCO 6000-8000) for 4 days with several water changes, and finally went to freeze-drying step.

\section{Preparation of BCP}

BCP-NPs were synthesized by using an ultrasonic assisted process. The calcium chloride reacted to tricalcium phosphate salts with molar ratio of $\mathrm{Ca} / \mathrm{P}=1.53$ for $12 \mathrm{~h}$ at $50^{\circ} \mathrm{C}$ under controlled $\mathrm{pH} 7$ to obtain a white suspension. The precipitate was washed thoroughly with DI water and dried in an oven at $70^{\circ} \mathrm{C}$. Finally, the calcination was carried out at $750^{\circ} \mathrm{C}$ in air. 


\section{Characterization of BCP}

The BCP product was X-ray diffraction analysis on D8/Advance XRD, Bruker conditions as follows: radiation wavelength $\mathrm{Cu}-\alpha=1.5406 \times 10-10 \mathrm{~m}$, scanning angle $2 \theta=10-60^{\circ}$, step size $0.02^{\circ}$ sec.

\section{Fabrication of hydrogel composite}

The hydrogel composite was attained by blending oxidized alginate (10\%wt) with gelatin (10\%wt) and various BCP weight ratio of BCP: polymers, namely, 0:100; 10:90; 30:70 in the PBS, with the assistance of the magnetic stirrer. The gelling time noted as the time required for the stir bar to stop. The cross-linking was formed between the chemical coupling of gelatin and oxidized alginate base on the Schiff-base reaction.

\section{Degradation of hydrogel composites}

The degradation of hydrogel composites was studied in PBS. The hydrogel composites were lyophilized and weighed $\left(W_{0}\right)$. These lyophilized hydrogel composites were immersed in $10 \mathrm{~mL}$ PBS solution at $37^{\circ} \mathrm{C}$. After regular time intervals, water was removed from the samples and washed with deionized water to remove the soluble inorganic salt then weighted $\left(W_{t}\right)$ after lyophilization. The percentage of weight loss was calculated to evaluate the degradation of hydrogel composite as following formula:

$$
\text { Weight loss }(\%)=\frac{W_{0}-W_{t}}{W_{0}} \times 100 \% \text {. }
$$

\section{Evaluation of bio-mineralization and bio-degradation of the material}

To study the possible precipitate phase conversion, hydrogel composite samples were immersed in a SBF buffer solution ( $\mathrm{pH}$ 7.4). Hydrogel composite was prepared and then lyophilized. Lyophilized hydrogel composite was cut to observe spongy surface and its weight was then recorded. Hydrogel composite was collected after 2 weeks soaking in SBF solution and then washed with deionized water to remove the 
soluble inorganic salt. Finally, the product was characterized by scanning electron microscopy (SEM), energy dispersive X-ray spectroscopy (EDS), and X-ray diffraction (XRD) methods. The concentration of calcium, phosphate ions was evaluated by inductively coupled plasma atomic emission spectroscopy (ICP-AES).

\section{Cell proliferation study}

Firstly, $5 \times 10^{4}$ mesenchymal stem cells derived from rabbit bone marrow cells seeded onto the UV-sterilized samples in 24-well plates for incubation, and came up with washing step by PBS for three times. The cell nuclei were counterstained with $20 \mathrm{mg} / \mathrm{mL}$ DAPI for $10 \mathrm{~min}$ at $\mathrm{RT}$, the sample then washed 3 times with $1 \mathrm{X}$ PBS. Finally, confocal laser scanning microscope (FV10i-W) was conducted to observed the stained cells on hydrogel composites after 1, 3, 5 days of cell seeding.

\section{Animals and surgical procedure}

Bone tissue engineering experiments using male New Zealand rabbits (6 months old) were carried out according to the guidelines provided by laboratory of stem cell research \& application, University of Sciences, Ho Chi Minh city, Vietnam. Each rabbit was anesthetized before the operation. Rabbits were randomly assigned into 3 groups ( $n=$ 3 for each group): untreated group (without implantation), hydrogel treatment group, hydrogel composite treatment group. The defect size was $5 \mathrm{~mm}$ diameter, $5 \mathrm{~mm}$ depth which was created in the femur. In each group, rabbits were sacrificed at 4 months.

\section{Radiological evaluation}

Each femur was scanned with a model 1076 X-ray micro-CT, scanning was carried out at pixel size of $9 \mu \mathrm{m}$. Image data are analyzed by using the SkyScan software (SkyScan; Kontich, Belgium) that displays the data as two-dimensional (2D) axial, sagittal, and coronal crosssectional images. 


\section{Statistical analysis}

ANOVA statistical analysis was performed on the data to determine statistical significance of the different sub populations. This process was accomplished using the data analysis package added to excel (Microsoft, Seattle, WA). All trials unless otherwise indicated had at least a population of 3. Statistical significance was rated at $p<0.05$.

\section{Results and Discussion}

Weight loss in a buffer solution is usually used to evaluate the degradation rate of hydrogel composite in vitro following a function of time. The requirement of bone graft materials is the initial structural stability and completely replacement by new bone over a desired time. This means the bone graft materials need a critical design in order to control the extent of biodegradation. As shown in Figure 1, all scaffolds can be degraded in physiological medium, and their degradation rate decreases with the increase of BCP content in hydrogel composite. After 4 weeks soaking in physiological medium, the weight loss of hydrogel composite free $\mathrm{BCP}$, hydrogel composite containing $10 \% \mathrm{BCP}$, and hydrogel composite containing $30 \% \mathrm{BCP}$ were $32.6 \%, 31.5 \%$, and $29.7 \%$, respectively. It is reasonable that the degradation rate of oxidized alginate-gelatin hydrogel can be controlled by changing the BCP content in hydrogel composite for varying application. This can be explained by the interaction of BCP particle and gelatin in hydrogel composite. Gelatin backbone comprising various functional groups such as $\mathrm{NH}_{2}, \mathrm{OH}$, and $\mathrm{COOH}$ can perform hydrogen bonding with $\mathrm{OH}$ group of HAp in BCP. In addition, the amine groups in gelatin can bind to $\mathrm{Ca}^{2+}$ in $\mathrm{BCP}$ to form the complex [14, 15]. Furthermore, the hydrolytic susceptibility of crosslinking between gelatin and oxidized alginate would capable to be completely degraded into low molecular weight polysaccharides and polypeptides, therefore, no harmless byproducts can be processed by the body. 


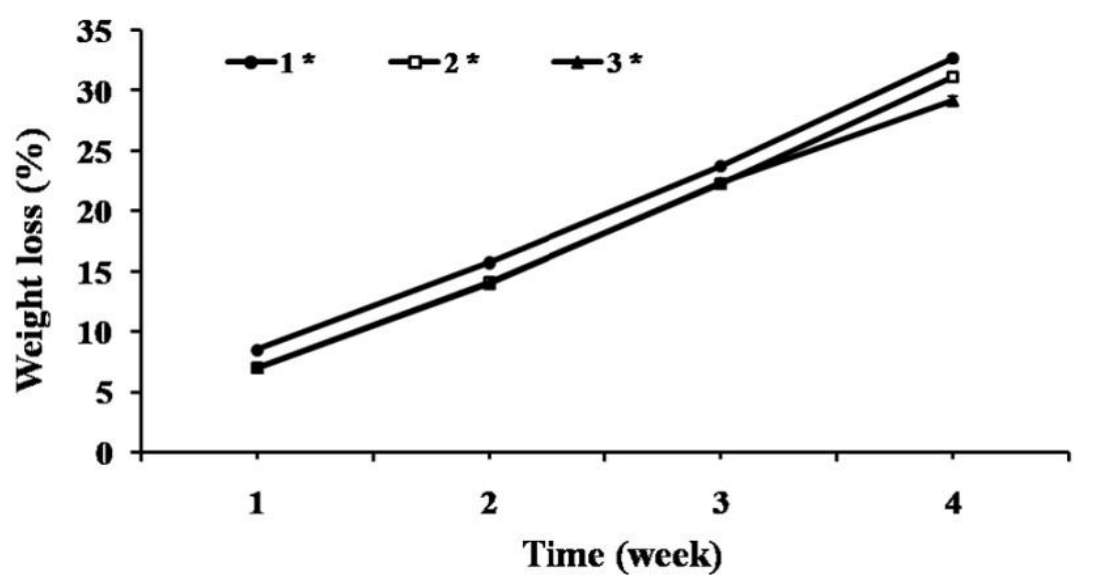

Figure 1. Degradation in weight (\%) of oxidized alginate-gelatin/BCP hydrogel composite: (1) 0\% BCP, (2) 10\% BCP, (3) 30\% BCP, ${ }^{*} p<0.05$.

Bone regeneration strategies realize the priority of the biomaterials able to mineralize by which native apatite crystals are formed in vivo. The bone-bonding ability of biomaterial was evaluated by the formation of the apatite layer on the surface of biomaterial immersed in the SBF solution [16]. In this study on inducing deposition of minerals on hydrogel composites, the driving idea was soaking in the SBF solution for up 1 week in order to predict the achievement of nucleation of apatite on their surface using SEM/EDS micrographs and XRD profiles.

As shown in SEM images of lyophilized oxidized alginate-gelatin hydrogel (Figure 2) the surface morphology exhibits some flake formation. According to the EDS analysis, the flake formation on the surface of hydrogel is the presentation of $\mathrm{Na}, \mathrm{Cl}$ elements. The resulting layer on the surface of hydrogel immersed in SBF solution indicated hydrogel has the poor mineralization. 

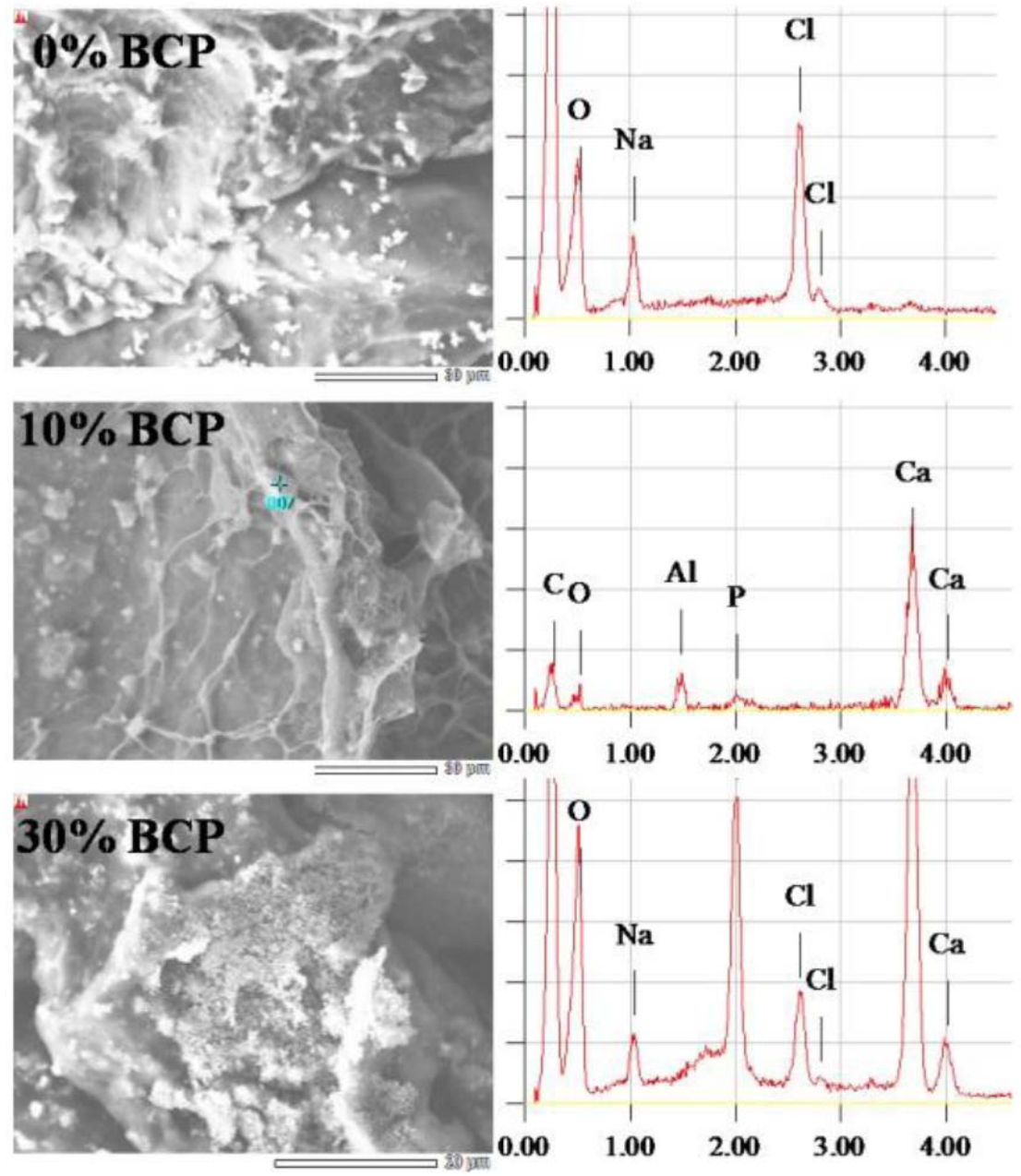

Figure 2. SEM images and EDS profile of oxidized alginate-gelatin/BCP hydrogel composites after 7 days soaked in SBF solution.

In case of oxidized alginate-gelatin-BCP hydrogel composites, the SEM images (Figure 2) indicate crystal particles on the surface of hydrogel composites. EDS analysis on these crystal particles (Figure 2) shows $\mathrm{C}, \mathrm{O}, \mathrm{P}$, and $\mathrm{Ca}$ elements indicates that the nucleated crystals are calcium phosphate and calcium carbonate, implying that initial deposition of mineral phases occurred, which could be further confirmed by XRD analysis (Figure 3). 


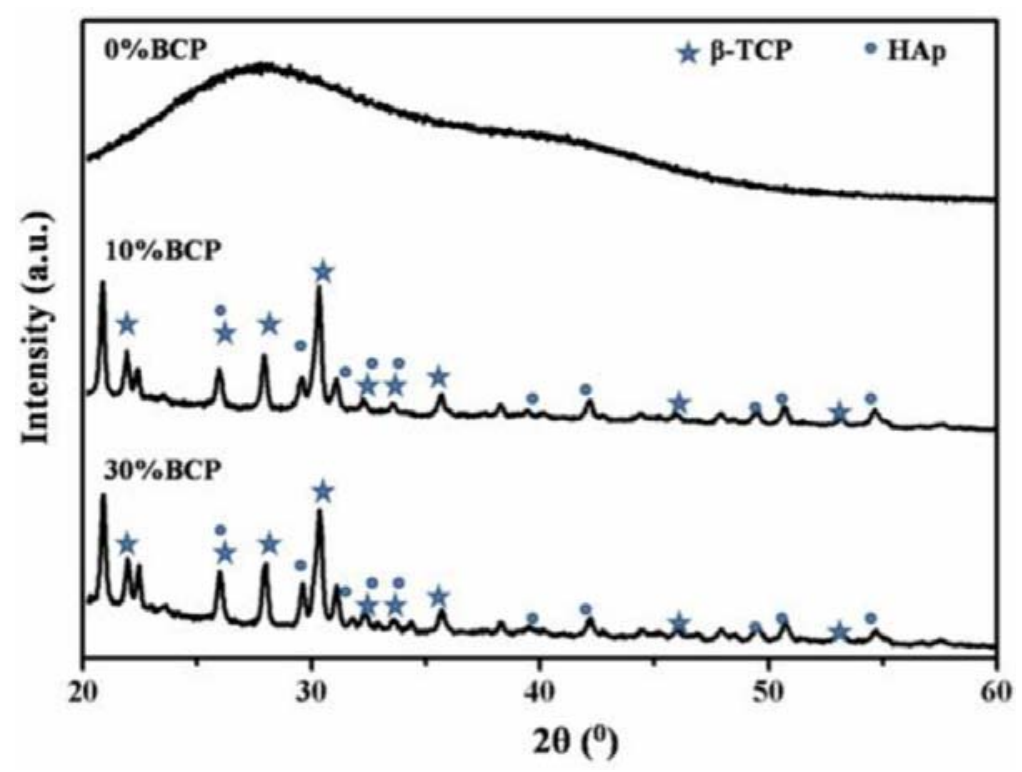

(a)

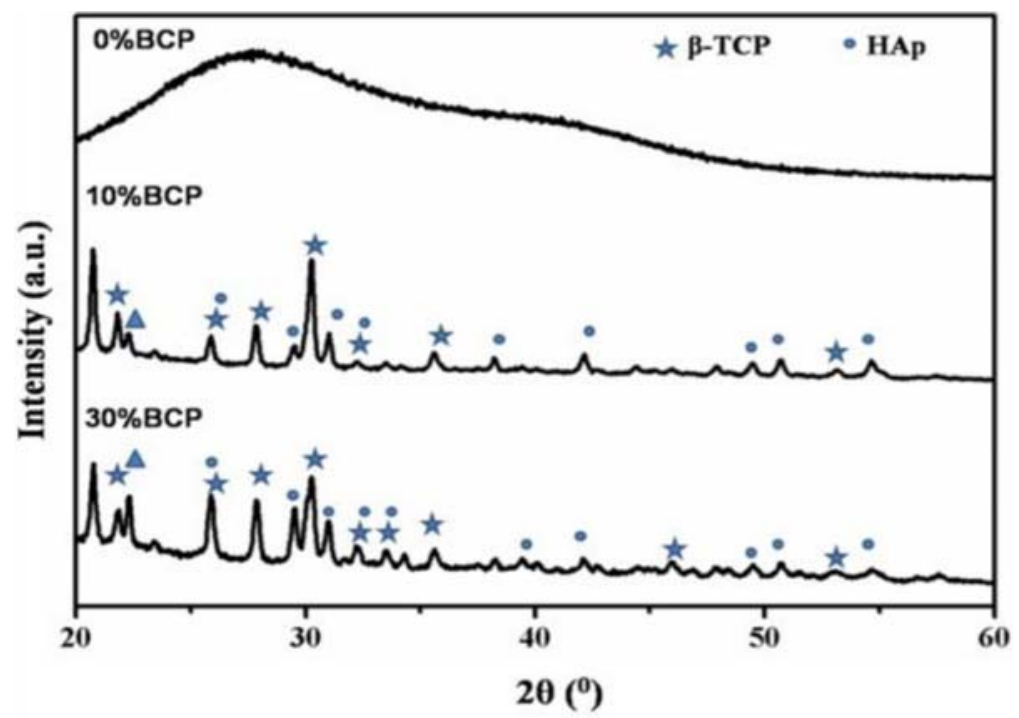

(b)

Figure 3. XRD profile of hydrogel composite oxidized alginate-gelatin/BCP hydrogel composite after soaking in SBF 0; 7days. 
The XRD profiles of hydrogel composite and hydrogel composite immersed in SBF solution is showed in Figure 3. The two peaks at 31.6 and 45.3 in the XRD profiles of oxidized alginate-gelatin-BCP hydrogel composite belong to the crystalline phase of alginate. The profiles show that the crystallinity of both the organic and the inorganic phase were retained in the hydrogel composite. No change was observed after SBF immersion (Figure 3) which explains that the precipitation on the surface of hydrogel composites was apatite.

Figure 4 shows that the concentration of calcium, phosphate ions recorded for oxidized alginate-gelatin hydrogel, oxidized-gelatin-10wt\% $\mathrm{BCP}$, and oxidized alginate-gelatin-30wt\% BCP hydrogel composite. 


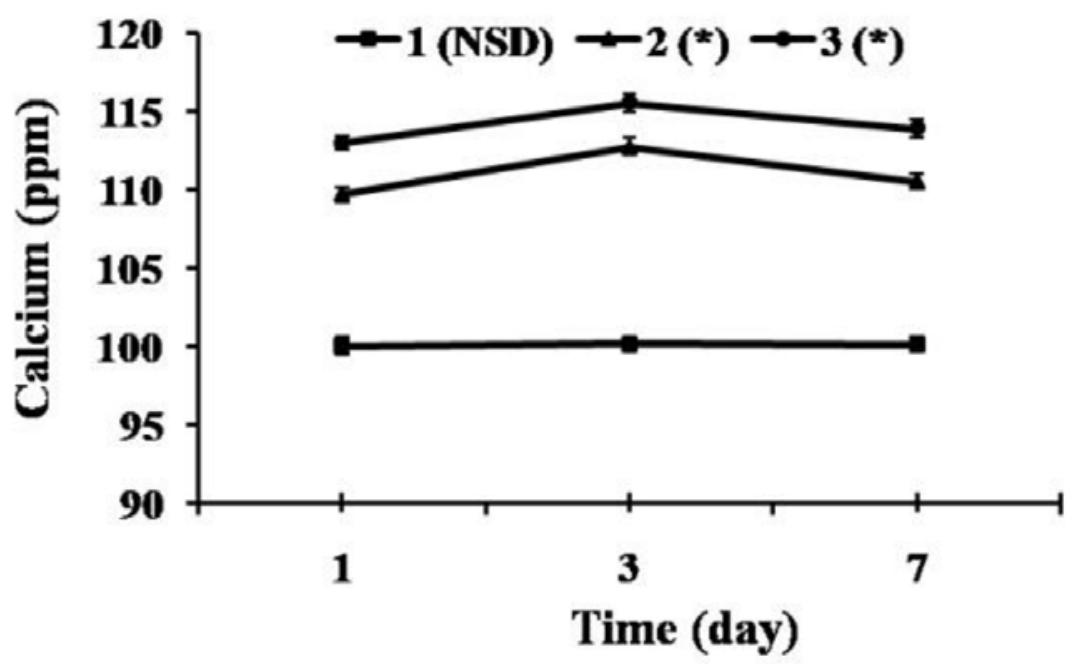

(a)

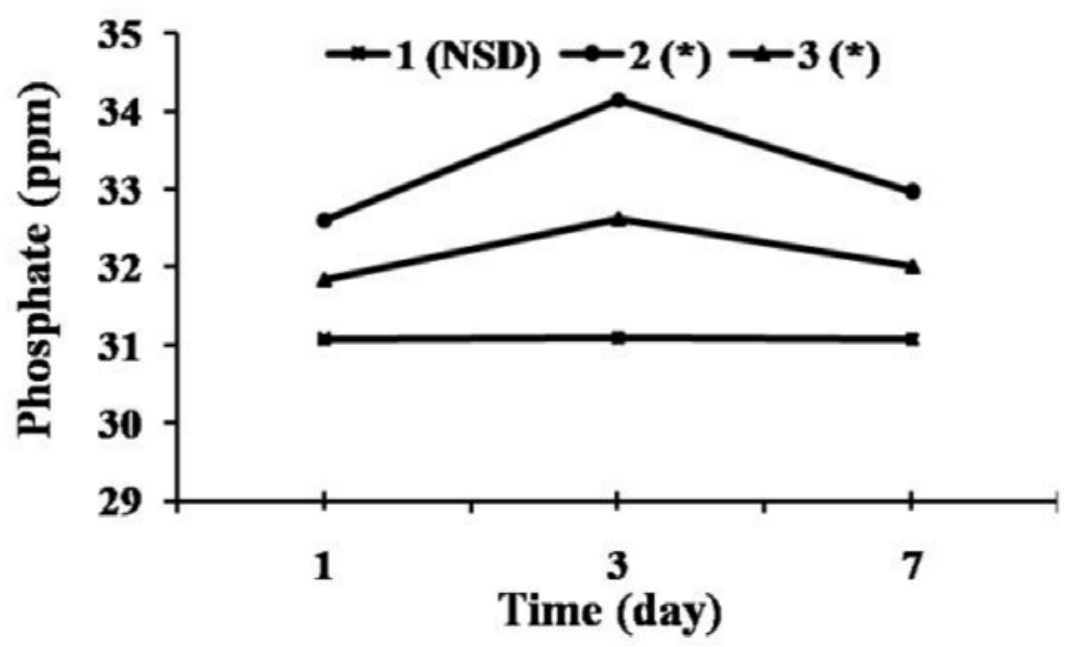

(b)

Figure 4. The calcium and phosphate ion concentration change of the samples after immersion in a SBF solution as a function of soaking time. (1) $0 \% \mathrm{BCP}$, (2) $10 \% \mathrm{BCP}$, (3) $30 \% \mathrm{BCP},{ }^{*} p<0.05 \mathrm{NSD}$ : Non significant difference, ${ }^{*} p<0.05$. 
In case of hydrogel, no significant change in the concentration of calcium and phosphate ions was observed. When hydrogel composites were immersed in the SBF solution, the concentration of these ions in the SBF solution increased in the first 3 days soaking but decreased gradually as the soaking period increased. This reveal the enhancement of calcium and phosphate ions concentration gradient after 3 days soaking is due to be the BCP dispersion. After immersion in the SBF solution 7 days, suggestion that the decrease in concentration of these ions is the results of a phenomenon of precipitation of numerous tiny flake to the surface of hydrogel composite which observed in SEM images.

The reason for the increase in calcium concentration during this phase was the dissolution behaviour of BCP in the composite gradually dissolved and released calcium and phosphate ions which benefit the formation of apatite. Moreover, the hydroxyl and phosphate unit in HAp, TCP crystal structure revealed a negative charge of BCP particle surface when hydrogel composite immersed in SBF. This negative charge attracted the positive calcium ions in SBF to form a rich calcium surface, which interacts with the negative phosphate ions in SBF. Calcium concentration was changed because $\mathrm{SBF}$ was supersaturated and any ionic dissociation in the SBF would break this equilibrium. As a consequence, calcium and phosphate ions migrated to surface of hydrogel composite that induced the apatite precipitation [17, 18].

The new phase deposited on the surface of hydrogel composite was apatite. This result was proved by SEM, XRD patterns as shown in Figure 3. For hydrogel composite, apatite was observed as samples immersed in SBF solution. The results indicate that BCP enhanced the apatite forming ability on composites. BCP particles act as apatite nucleation sites, and then the apatite nuclei could grow by the absorption of calcium and phosphate ions from the SBF solution. Therefore, the formation of apatite is more efficient on the composite with higher content of BCP. The apatite precipitation through SBF test was a consequence of a dissolution and precipitation process of calcium 
phosphate. These observations claimed that new apatite layer could rapidly from after the soaking of this hydrogel composite scaffolds in the SBF solution, which is another expression of bioactivity in vitro.

Evaluation on adhesion and proliferation of mesenchymal stem cells derived from rabbit bone marrow on hydrogel and hydrogel composites was observed under fluorescence microscope (Figure 5). The results show that mesenchymal stem cells derived from rabbit bone marrow well adhered on the surface of hydrogel and hydrogel composites. After 5 days, mesenchymal stem cells derived from rabbit bone marrow were coated on their surface. It may be explained that gelatin and BCP stimulate cell development. The positive influence of BCP on the behaviour of cells has been reported such as high adsorption capacity for serum protein, enhanced cellular attachment [19-22].

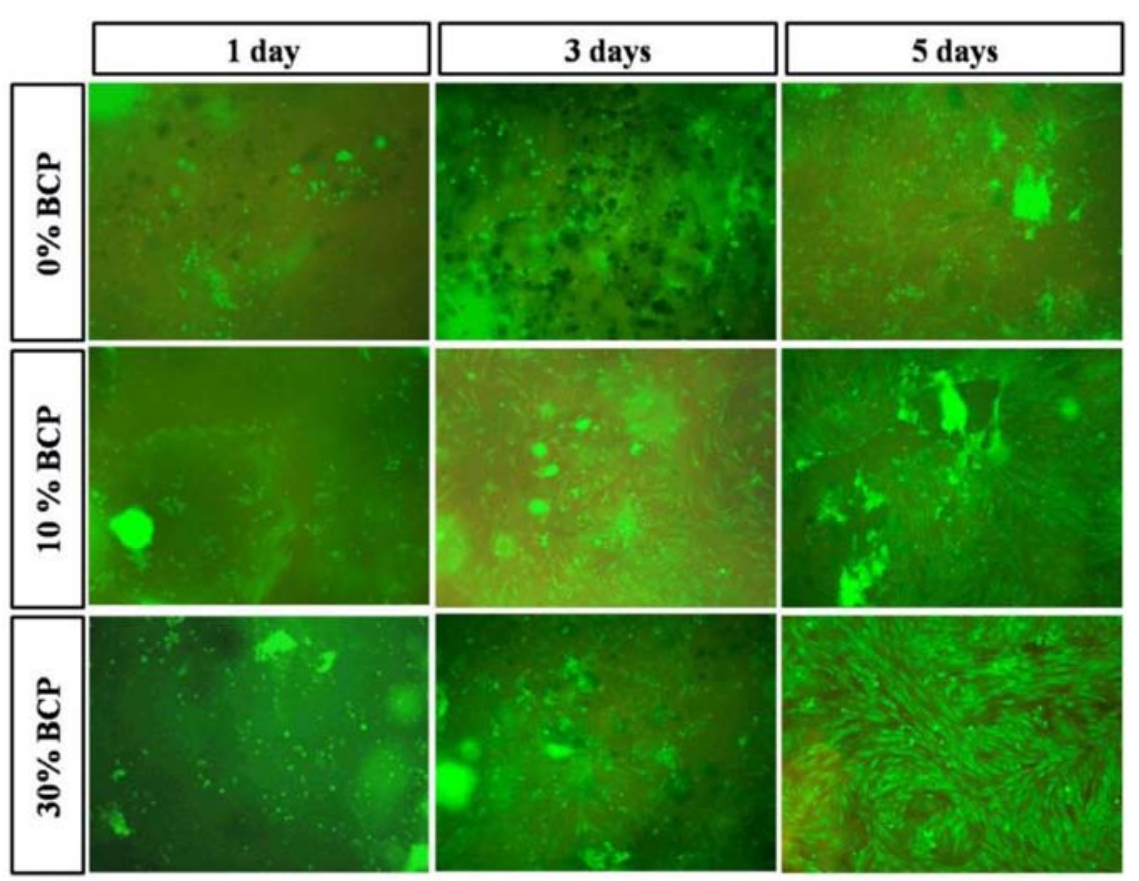

Figure 5. Confocal images of mesenchymal stem cells derived from rabbit bone marrow adhesion on oxidized alginate-gelatin-BCP hydrogel composites, depending on BCP contents and cell incubation times. 
The bone formation can be determined from the micro-CT analysis. The ability of the hydrogel composite to facilitate bone growth within bone defect was examined in the rabbit femur defect by using the micro-CT.

The morphometric parameter (BV/TV) using CT analysis was shown in Figure 6. Quantification of newly formed bone showed that the percent bone volume (BV/TV\%) was $(8.37 \pm 0.14) \%$ in control, $(8.01 \pm 0.37) \%$ in hydrogel scaffolds, and $(13.28 \pm 1.58) \%$ in hydrogel composite after 4 months of implantation, a higher amount of bone formation was observed in hydrogel composite scaffolds than in hydrogel scaffolds and control. In the orthotopic sites, bone formation stared at the layer interface between scaffold-host bone, BCP has positive effects to migratation of osteoblast cells, and differentiation of osteoblasts resulted in increased bone formation in hydrogel composite than hydrogel [23-26].

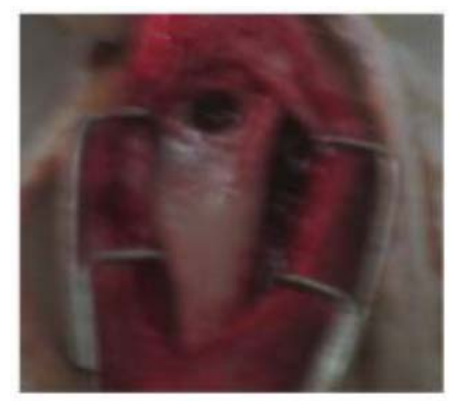

(a)

\section{Control}

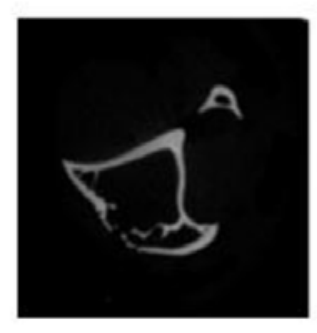

Hydrogel

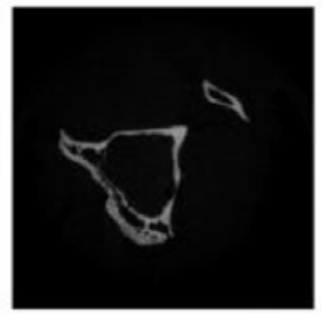

Hydrogel composite

(b)

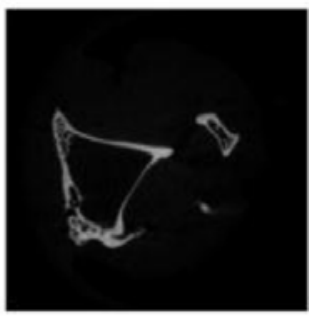




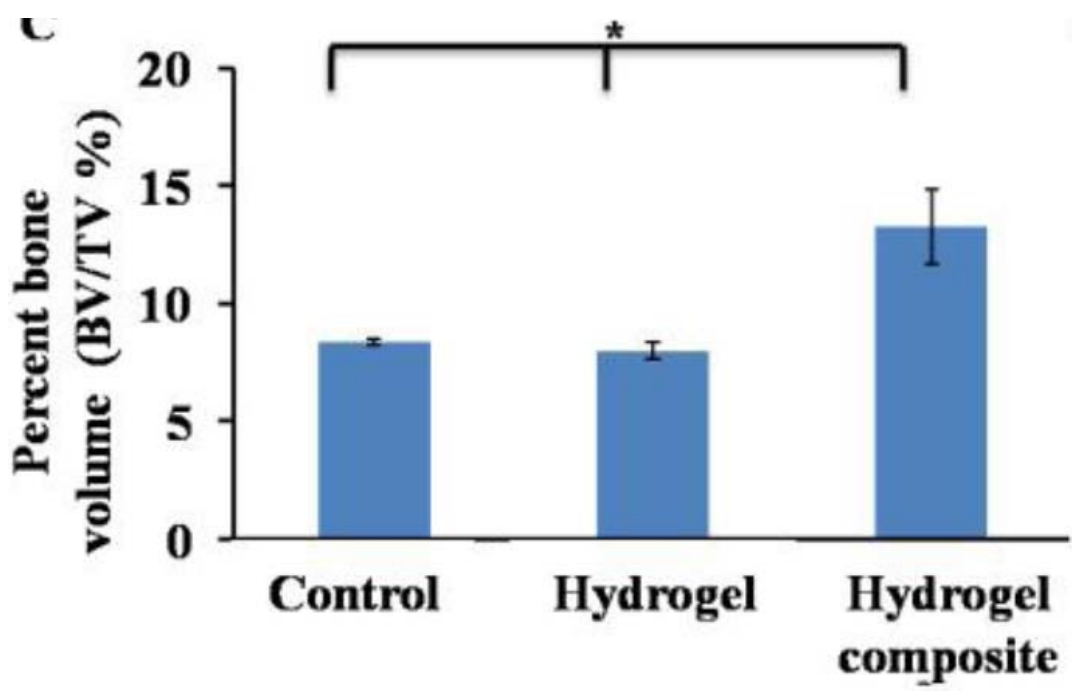

(c)

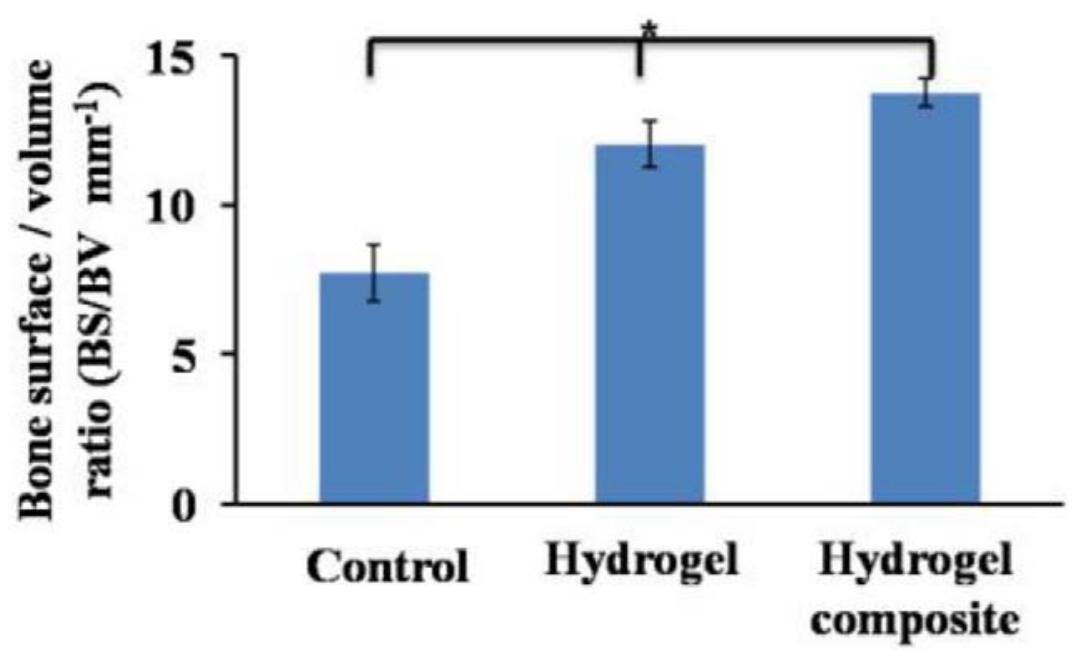

(d)

Figure 6. (A) Surgery placement in femur, (B) micro-CT images of the graft in femur defects at 4 months post implant, (C) quantitative analysis of percent bone volume (BV/TV\%) at the defect sites at 4 months of implantation, and (D) quantitative analysis of bone surface/volume ratio $\left(\mathrm{BS} / \mathrm{BV} \mathrm{mm}^{-1}\right)$ the defect sites at 4 months of implantation, ${ }^{*} p<0.05$. 
Bone surface/ bone volume ratio in hydrogel composite group (13.78 \pm $0.49)$ and hydrogel group $(12.02 \pm 0.76)$ was higher than control group $(7.73 \pm 0.95)$. This reveals the interaction between the surface of scaffold and cell, in which cell can proliferate, thus, it is able to enhance the calcified tissue. In the control group, marrow cavity is degraded, but not in the scaffold cases, bone was reconstructed based on the marrow cavity of the amputation site [27]. Therefore, the density surface is higher in animal with scaffolds than the control group.

\section{Conclusion}

In summary, this study BCP loaded in oxidized alginate-gelatin hydrogel has the advantages of biomineralization, cell adhesion, and bone formation ability. As BCP content increased, the proliferation of cells on the surface of hydrogel composites increased. BCP enhanced the apatite forming ability on composites, and the bone healing process. Hence, oxidized alginate-gelatin/BCP hydrogel composite can be considered for potential application in bone regeneration.

\section{Acknowledgements}

This study was supported by the grants of Department of Science and Technology Ho Chi Minh City, Vietnam, contract number 359/2013/HĐSKHCN.

\section{References}

[1] M. Navarro, A. Michiardi, O. Castaño and J. A. Planell, Biomaterials in orthopaedics, Journal of the Royal Society Interface 5 (2008).

[2] A. S. Hoffman, Hydrogels for biomedical application, Advanced Drug Delivery Reviews 43 (2002), 3-12.

[3] K. Gkioni, S. C. Leeuwenburgh, T. E. Douglas, A. G. Mikos and J. A. Jansen, Mineralization of hydrogels for bone regeneration, Tissue Engineering Part B Reviews 16 (2010), 577-585.

[4] W. Cao and L. L. Hench, Bioactive materials, Ceramics International 22 (1996), 493. 
[5] S. M. Rea, S. M. Best and W. Bonfield, Bioactivity of ceramic-polymer composites with varied composition and surface topography, Journal of Materials ScienceMaterials in Medicine 15 (2004), 997.

[6] N. Engin and A. Tas, Manufacture of macroporous calcium hydroxyapatite bioceramics, Journal of the European Ceramic Society 19 (1999), 2569-2572.

[7] D. Michel, B. Laurent, M. Gérard, T. Arnaud, L. Jianxi, L. Anne, L. Véronique and C. Francis, Processing and properties of biphasic calcium phosphates bioceramics obtained by pressureless sintering and hot isostatic pressing, Journal of the European Ceramic Society 33 (2013), 1263-1270.

[8] S. T. P. Manjubala and R. V. S. Kumar, Bone in-growth induced by biphasic calcium phosphate ceramic in femoral defect of dogs, Journal of Biomaterials Applications 19 (2005), 341-360.

[9] K. Anselme, Osteoblast adhesion on biomaterials, Biomaterials 21 (2000), 667-681.

[10] V. Sanginario, M. P. Ginebra, K. E. Tanner, J. A. Planell and L. Amabrosio, Biodegradable and semi-biodegradable composite hydrogels as bone substitutes: Morphology and mechanical characterization, Journal of Materials ScienceMaterials in Medicine 17 (2006), 447-454.

[11] K. R. Mohamed, H. H. Beherei and Z. M. El-Rashidy, In vitro study of nanohydroxyapatite chitosan gelatin composites for bio applications, Journal of Advanced Research 5(2) (2014), 201-208.

[12] P. Wang, L. Zhao, J. Liu, M. D. Weir, X. Zu and H. H. K. Xu, Bone tissue engineering via nanostructured calcium phosphate biomaterials and stem cells, Bone Research 2 (2014), 14017.

[13] T. P. Nguyen and B. T. Lee, Fabrication of oxidized alginate-gelatin-BCP hydrogels and evaluation of the microstructure, material properties and biocompatibility for bone tissue regeneration, Journal of Biomaterials Applications 27(3) (2012), 311-321.

[14] Y. Yuji, Y. Fen, C. Junfeng, Z. Fujing, L. Xiulan and Y. Kangde, Preparation and characterization of macroporous chitosan-gelatin/b-tricalcium phosphate composite scaffolds for bone tissue engineering, Journal of Biomedical Materials Research 67A (2003), 844-855.

[15] L. Junjie, D. Yan, Y. Jun, Y. Yuji, Z. Hong, Y. Fanglian, W. Haibin and Y. Kangde, Surface characterization and biocompatibility of micro- and nano-hydroxyapatite/ chitosan-gelatin network films, Materials Science and Engineering C 29 (2009), 1207-1215.

[16] A. Veis and J. R. Dorvee, Biomineralization mechanisms: A new paradigm for crystal nucleation in organic matricies, Calcified Tissue International 93(4) (2013), 307-315.

[17] T. Kokubo and H. Takadama, How useful is SBF in predicting in vivo bone bioactivity?, Biomaterials 27(15) (2006), 2907-2915. 
[18] A. A. Zadpoor, Relationship between in vitro apatite-forming ability measured using simulated body fluid and in vivo bioactivity of biomaterials, Materials Science and Engineering C- Materials for Biological Applications 35 (2014).

[19] K. Anselme, Osteoblast adhesion on biomaterials, Biomaterials 21(7) (2000), 667-681.

[20] J. Venugopal Gupta, S. Mitra, V. R. G. Dev and S. Ramakrishna, Nanostructured biocomposite substrates by electrospinning and electrospraying for the mineralization of osteoblasts, Biomaterials 30(11) (2009), 2085-2094.

[21] M. Ngiam, S. Liao, A. J. Patil, Z. Cheng, C. K. Chan and S. Ramakrishna, The fabrication of nano-hydroxyapatite on PLGA and PLGA/collagen nanofibrous composite scaffolds and their effects in osteoblastic behavior for bone tissue engineering, Bone 45(1) (2009), 4-16.

[22] L. Ambrosio Ciapetti, L. Savarino, D. Granchi, E. Cenni, N. Baldini, S. Pagani, S. Guizzardi, F. Causa and A. Giunti, Osteoblast growth and function in porous poly $\varepsilon$-caprolactone matrices for bone repair: A preliminary study, Biomaterials 24(21) (2003), 3815-3824.

[23] L. Zhang, N. Hanagata, M. Maeda, T. Minowa, T. Ikoma, H. Fan and X. Zhang, Porous hydroxyapatite and biphasic calcium phosphate ceramics promote ectopic osteoblast differentiation from mesenchymal stem cells, Science and Technology of Advanced Materials 10(2) (2009).

[24] A. L. Gamblin, M. A. Brennan, A. Renaud, H. Yagita, F. Lézot, D. Heymann, V. Trichet and P. Layrolle, Bone tissue formation with human mesenchymal stem cells and biphasic calcium phosphate ceramics: The local implication of osteoclasts and macrophages, Biomaterials 35(36) (2014), 9660-9667.

[25] N. Özkucur, T. K. Monsees, S. Perike, H. Q. Do and R. H. W. Funk, Local calcium elevation and cell elongation initiate guided motility in electrically stimulated osteoblast-like cells, PLoS One 4(7) (2009), e6131.

[26] S. Maeno, Y. Niki, H. Msumoto, H. Morioka, T. Yatabe, A. Funayama, Y. Toyama, T. Taguchi and J. Tanaka, The effect of calcium ion concentration on osteoblast viability, proliferation and differentiation in monolayer and 3D culture, Biomaterials 26(23) (2005).

[27] A. Golding, J. A. Guay, C. H. Rincon, M. Levin and D. L. Kaplan, A tunable silk hydrogel device for studying limb regeneration in Adult Xenopus Laevis, PLoS One 11(6) (2016), e0155618. 